\title{
Green Business Process Patterns
}

\author{
Alexander Nowak \\ nowak@iaas.uni-stuttgart.de
}

\author{
Frank Leymann \\ leymann@iaas.uni-stuttgart.de
}

\author{
Daniel Schleicher \\ schleicher@iaas.uni-stuttgart.de
}

\author{
David Schumm \\ schumm@iaas.uni-stuttgart.de
}

\author{
Sebastian Wagner \\ wagner@iaas.uni-stuttgart.de
}

\author{
Institute of Architecture of Application Systems (IAAS) \\ University of Stuttgart \\ Universitaetsstrasse 38, 70569 Stuttgart, Germany
}

\begin{abstract}
To ensure their competitive advantage an increasing number of organizations adopt business process management for design, automation, and analysis of their business processes. In order to reduce cost, improve quality, save time, and increase flexibility, techniques for business process improvement and re-engineering are applied. Improving the environmental impact of a business process is a new challenge organizations are faced with. However, current approaches and techniques for business process optimization do not cover the ecological dimension explicitly. In this paper, we propose patterns which describe good solutions for green business process design to address this gap from a business perspective. The patterns are described independently from concrete business process modeling languages and execution environments in order to provide a broad applicability of the patterns within different scenarios. In addition to the patterns, we discuss the general usability of the patterns based on different aspects relevant to an organization.
\end{abstract}

\section{Keywords}

Green IT, Green Patterns, Green Business Process Management, Environmental Impact

\section{INTRODUCTION}

Business Process Management (BPM) refers to concepts, methods, and techniques to support the design, administration, configuration, enactment, and analysis of business processes [1]. Through the application of BPM, organizations try to realize cost reductions, quality improvements, time savings, and increased flexibility [2]. In recent years, the concepts and methods of BPM have been extended in order to account for cross-cutting concerns. Besides cross-cutting concerns like security or compliance, one challenge is the proper application of BPM in the context of Green IT [3][5].

Permission to make digital or hard copies of all or part of this work for personal or classroom use is granted without fee provided that copies are not made or distributed for profit or commercial advantage and that copies bear this notice and the full citation on the first page. To copy otherwise, or republish, to post on servers or to redistribute to lists, requires prior specific permission and/or a fee.

PLoP'11, October 21-23, 2011, Portland, OR, USA.

Copyright 201a ACM 1-58113-000-0/00/0010 ...\$10.00.
The so-called Green BPM deals with the optimization of business processes based on the four common dimensions cost, quality, time, flexibility, and in particular also considers a fifth dimension: the ecological dimension. This dimension covers a variety of aspects like energy consumption, water consumption, or production waste incurring during performance of business processes and activities contained therein, respectively. Although Green BPM is still in an early stage, more and more organizations try to find different ways for improving the environmental impact of their business processes. The drivers for this development are manifold: increasing costs for raw-materials and energy, legislative regulations to reduce emission of carbon, or the public image of an organization, to name some examples. The main difficulty organizations are faced with is finding a proper solution for green business process improvement considering (a) the particular business process structure, (b) if and how the process may be changed, and (c) how the process interacts with business partners.

Current approaches for business process optimization mainly focus on the optimization of the four common performance dimensions. However, these approaches do not consider the ecological dimension explicitly. In [2], for example, the authors present a variety of ways to address these regular dimensions. As a side-effect, the optimization of the common dimensions also has a positive impact on the ecological dimension in most cases. We argue that there are ways beyond the common forms of business process improvement which consider the ecological dimension explicitly. In order to also address this dimension as an aspect for process optimization, we propose to use a pattern approach for describing appropriate solutions for the ecological optimization of business processes.

The main contribution of this paper is a set of green business process patterns. These patterns follow the guidelines and examples of pattern languages [7][8][16][22] and address the green (re-)design of business processes from an abstract perspective. They are independent from a specific BPM environment or process modeling language. Through this abstraction, organizations can apply these patterns to their individual BPM environment which, however, needs an in-depth analysis of the concrete scenario. The application of multiple patterns is furthermore possible. Another contribution of the paper is an analysis of the patterns we have identified. In this analysis 
we provide a decision tree and classification that helps decision makers to identify relevant patterns. We also critically discuss usage scenarios of some patterns. We argue, that by applying the proposed patterns organizations are able to identify, define and realize custom implementations referring to a specific process adaptation using their available techniques and tools.

The remainder of this paper is structured as follows: Section 2 describes the background and the approach used for identifying the patterns and their structure. The section further contains the identified patterns sorted by our proposed classification, which is presented in Section 3. This section also includes an analysis and decision support for choosing an appropriate pattern with respect to different aspects relevant to organizations. A critical discussion of the usage of the patterns is presented in Section 4. Section 5 then concludes this paper.

\section{GREEN BUSINESS PROCESS PATTERNS}

In this section we present frequently occurring forms of green business process design which we identified in the offerings of several companies and in recent research in the field of Green IT. In these works, the patterns are applied implicitly to provide green products or green services by making the processes from which they originate "greener". In addition to these forms of green business process design, we investigated the regular forms of process (re-)design with focus on their applicability in the context of improving the ecological dimension. As a result, we identified and explicated the forms of green business process design as green business process patterns. We argue that an abstraction of solutions to make processes greener is beneficial for the discussion and comparison of green BPM approaches. It also helps to formulate a strategy for green business process improvement and product marketing management. We do not claim that the list of patterns we present is complete - but it represents the result of our abstraction from product and service offerings [9][10][11][12][13][14][15], literature on BPM in general [1][2][17] and Green BPM in particular [3][4][5], and our own research in the field of Green IT and business process analysis [18][19][20].

Before we proceed with the description of the patterns we want to introduce the terminology used in this paper. A business process consists of a set of activities that are performed in coordination in an organization to jointly realize a business goal [1]. The design of a business process is captured in a business process model. This model consists of a set of activity models and execution constraints between them. Consequently, a business process instance represents a concrete case in the operational business of a company, consisting of activity instances [1]. Such an activity instance can be either performed automatically, semiautomatically or manually. Usually, the process flow, i.e. the predecessor and successor relationship between activities, is controlled only by the process owner who's executing the complete process on his own. In this case, the business processes is coordinated by a so-called orchestration which implements the process by expressing the order of as well as the data flow between the individual process activities. The main benefit of the use of orchestrations is the possibility to rapidly adapt the process to new business requirements by just changing single activities. However, business processes also play a major role in collaboration scenarios. In these settings each partner has full control over and responsibility for the execution of their own business processes. Provided that every partner implements their processes as orchestrations, complex conversations between these orchestrations occur. So-Called choreographies capture these conversations from a global perspective, i.e., internal activity invocations within one partner are hidden to the outside [23].

In order to perform a certain business process, activities are using resources that ensure the correct execution. Such resources can be raw-materials, a drill bit, a hard disk drive, electricity, or another process or service, for example. During its execution a business process has always a certain environmental impact. This comprises all aspects that influence the environment when executing the business process, like energy consumption, water consumption, $\mathrm{CO} 2$ emission, or waste. The basis for the calculation of these metrics is chosen by evaluating the aspects of interest of the execution of a business process. Energyconsumption, for example, can be measured with energy meters; the impact of waste can be represented by the proportion of harmful substances.

Within the proposed patterns we use the graphical representation of business processes described in Figure 1. Based on this notation, general activities are depicted as simple circles. If we want to emphasize specific types of activities we extend the standard notation and put a further symbol into the upper left corner of that circle. The predecessor and successor relationship between different activities is depicted by an arrow. We call these arrows control flows. In some cases, we are also interested in the resources of certain activities. These resources are exemplarily illustrated as computer symbols and are attached to activities via a dashed line. Following the context of this paper, business processes should be optimized with respect to their environmental impact. Thus, we depict the optimized processes, activities or resources by adding a little tree to their symbol and changing the color to a light green.

The pattern approach we are following in this paper is based on literature [16][21] on pattern writing. Consequently, as a common structure we provide a pattern name, a symbol for graphical representation, and a problem statement describing the problem to be solved with this pattern. We further provide the context in which the pattern applies, the challenges which the pattern addresses, a solution to tackle the challenges, a sketch depicting the solution in a graphical way (a legend of the symbols is depicted in Figure 1), the result of applying the pattern, an example of the application of the pattern, variations of the pattern usage, and the relationship to other patterns. We do not provide an explicit driver for each pattern as the drivers introduced in Section 1 apply to all patterns in the same way. Moreover, the choice of a particular pattern depends on the possible degree of restructuring of the business processes. The more the process can be modified, for example by substituting certain activities, the more patters may fit.

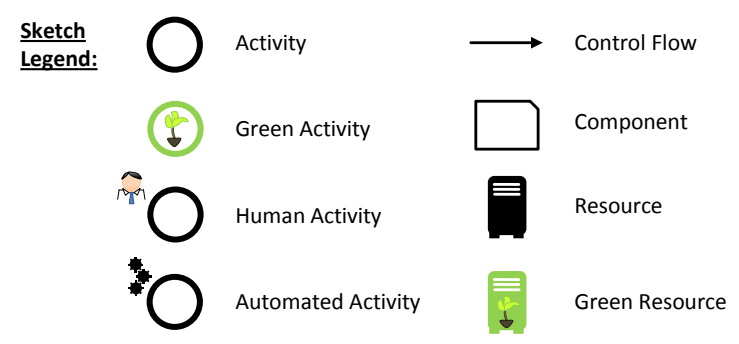

Figure 1 Legend for Pattern Sketches 
An important aspect regarding green business process design patterns is the trade-off between the existing key performance indicators (e.g., cost per instance) and the key ecological indicators representing the environmental impact of an activity or process (e.g., carbon emission per instance). When applying any of the proposed patterns one also has to deal with this trade-off. However, due to the abstract nature of the patterns the situation needs to be investigated for each and every scenario where the patterns are applied on. Thus, in this paper we do not go into detail for the specific applicability of each pattern in concrete scenarios.

\section{Pattern 1: Green Compensation}

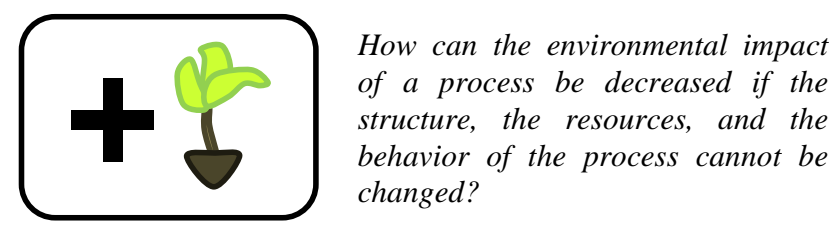

Context: Organizations have widely adopted business process management to manage and optimize their business processes. Within this domain organizations use business process design and analysis in order to identify potential optimization strategies. Some processes, however, may not be changed due to internal policies, laws, regulations or other constraints.

Challenges: Some processes within an organization may be hard to redesign or restructure due to the nature of tasks they are performing or due to other internal restrictions. The challenge is to find a proper solution for compensating the environmental impact without changing the process. In order to provide a trustworthy compensation, organizations should further implement a certified compensation alternative to improve visibility at the customer's site.

Solution: Whenever a business process that cannot be modified is initiated, a compensation process or activity is initiated, too. This process or activity compensates (parts of) the environmental impact caused by the original process.

Sketch:

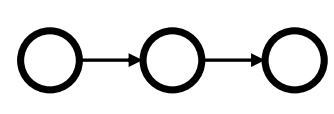

Original Process

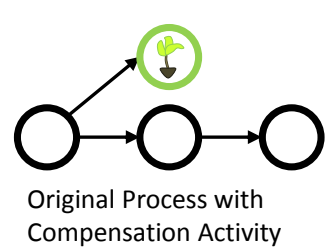

Compensation Activity
Results: Although the original process cannot be modified, the implementation of the pattern is able to achieve an improvement of the environmental impact from a global point of view because negative effects are not reduced, but compensated by positive effects. These positive effects may be further improved through an environmental certification of the corresponding compensation activities.

Examples: Production processes may require specific machines coming with a high upfront investment. These machines have certain well-defined process steps. The production waste originating from these machines, for example, may not be reduced without investing in new and expensive machines. So, although the production waste cannot be reduced, a donation for an environmental organization (e.g., for planting a tree or for buying rainforest) can be used to improve the environmental impact from a global perspective. Another example is the compensation of carbon emission occurred when burning fuel in auto mobiles. Oil companies provide a sustainability program that offers customers to compensate the amount of carbon dioxide for each refuel by investing in renewable energy.

Variations: This pattern can be used in a custom way by using conditional compensations. For example, the compensation process or activity is only performed at every tenth initiation of the original process. This decision can be based on different aspects like waste that has been produced during process performance or the compensation value, for example.

Relations to other Patterns: Depending on the type of business process it may be feasible to introduce a green variant as depicted in Pattern 2. The original process can then be initiated as usual while some instances could also base on the alternative process model that has less environmental impact.

\section{Pattern 2: Green Variant}

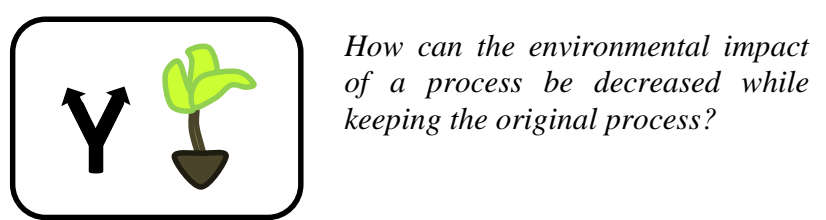

Context: Within the optimization of business processes, organizations may not change their business processes to a greener one without making them, for instance, more expensive or violating internal policies (green trade-off). Doing so would probably lead to a decrease in market share. However, organizations may be able to offer an alternative business process variant that has less environmental impact than the original one and gives the customers the opportunity to decide between the conventional and the green variant.

Challenges: In this case it is important to assure the same functional outcome of the process. Consequently, processes must be analyzed and restructured with respect to this objective, however, obtaining a decreased environmental impact.

Solution: The organization provides an alternative green business process variant that achieves the same result as before. However, it may use different process steps, resources, or partners to perform the process. The previous existing business process remains.

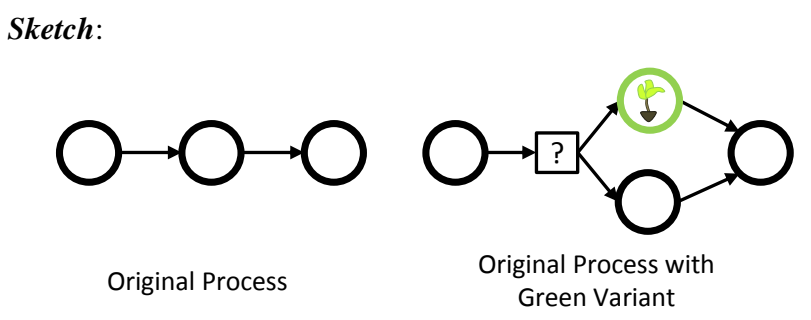

Results: Based on certain criteria like the customers decision, different variants of the process with the same functional outcome can be provided. Depending on which of the variants is being 
executed, different key performance indicators and ecological parameters hold.

Examples: A trading company usually uses conventional plastic or styrofoam to wrap their goods. Using this pattern, the company can offer an alternative packaging method, using non-plastic and renewable materials, e.g. made out of corn, to pack the goods. In case of increasing costs they can provide this offer as a customer's choice, i.e. customers decide whether they want conventional packaging or if they pay an extra charge for alternative packaging.

Variations: In the first place variations are achieved by conditioning the decision based on a certain criteria. Secondly, it would be possible to provide slightly different services or products. Depending on the business partners or customers choice, the service is performed differently (maybe with a different outcome) or products have another shape.

Relations to other Patterns: Depending on the type of business process it may also be feasible to implement a greener choreography (Pattern 8 and 9). In this case, it may also suffice to exchange certain business partners.

\section{Pattern 3: Resource Change}

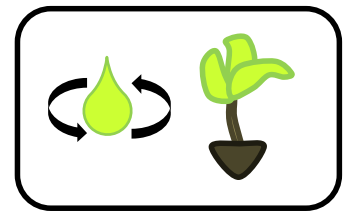

How can the environmental impact of a process be decreased while keeping the original process structure?

Context: Organizations are using a multitude of resources for the operation of their business and the corresponding business processes. These resources need to be selected based on both the strategic business and ecological objectives the organization wants to achieve.

Challenges: A resource is usually chosen by its functional capabilities. Selecting resources based on their environmental impact, however, can significantly influence the corresponding result. Additionally, proper metrics need to be defined and set-up in order to determine the different metric values of each resource.

Solution: Exchange the underlying resources of certain activities.

Sketch:

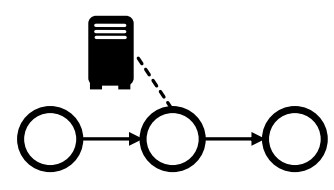

Original Process

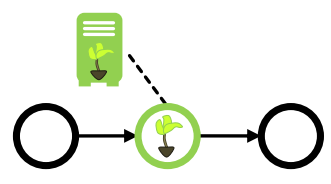

Original Process with Exchanged Resource
Results: Organizations will decrease their environmental impact by exchanging resources used by certain activities. The exchange of underlying resources usually does not affect the process structure. Thus, the process is performed as before, however, possibly with ecological-positive side-effects.

Examples: Resources are used or consumed in many cases. A company could, for example, decide to use green electricity instead of a conventional one. They may also decide to use different raw materials like non-plastic packaging for packaging their goods. Another example would be the use of cloud

computing. This decreases the number of machines that an organization needs to hold available (e.g. for rare peak situations) and allows a demand-dependent use of these resources.

Variations: Some resources display a different ecological impact based on other environmental factors, such as the form of available energy. A supplier of electric energy, for example, may provide greener electricity the windier it is at the location of his wind energy plant. This type of resource does not have to be exchanged but it is merely ensured that the same resource is used during certain time-slots in which its environmental impact is reduced.

Relations to other Patterns: Depending on the type of business process it may also be feasible to use Green Features described in Pattern 4. This pattern, however, may focus more on the appearance of a service or product rather than on an in-depth green process optimization.

\section{Pattern 4: Green Feature}

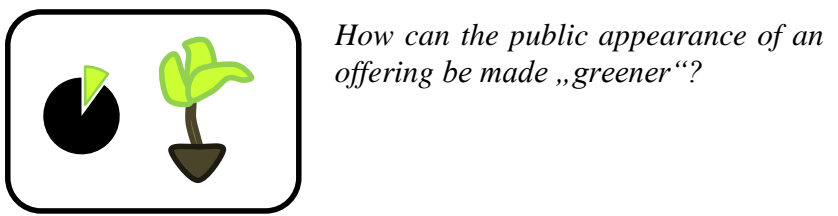

Context: Organizations gain a reputation of being green when they have at least a few outstanding green features in their products. A single feature may only contribute marginally to the overall ecological impact of their product. However, the combination of several different green features may significantly influence the environmental impact.

Challenges: Determine eye-catching features of a product which can be altered to be greener.

Solution: Identify all features of a product where a green alternative might be available. Replace those features with a greener alternative for which the greener alternative is reasonable from an economical point of view. One selection criterion should be the fact that it is easy to communicate to customers. Thus, this green feature can be used to attract the attention of the customers distracting from other less green features of the product.

Sketch:

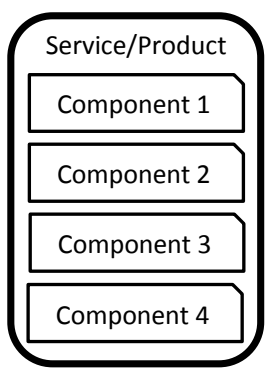

Original Components

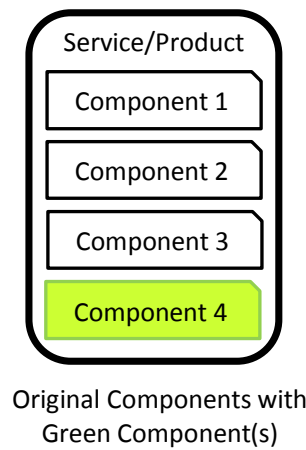

Results: A product which looks very ecofriendly to the customer although the impact of the green features of the product may only decrease the ecological footprint a little. 
Examples: An enterprise may advertise a production plant by showing a production process to the customers. This production process contains certain green features mentioned prominently, like specific activities which are executed on environmentally friendly machines. However, the remaining process is performed in a conventional way. Another example is the production of outdoor clothes. Most companies use the environmentally harmful fluorocarbon within the manufacturing process. Changing the manufacturing to be fluorocarbon free is a feature that can be used as competitive advantage compared to production of regular outdoor clothes. A similar example comes from the food industry. A lot of beverages come in plastic bottles made out of polyethylene terephthalate. A company in Europe adds specific syrup originating from sugar production to their bottles which reduces the share of polyethylene terephthalate.

Variations: Repeated application of this pattern may lead to a product comprising only of features considered green. In this case the products impact on the environment is improved even more. Another variant could be the environmental certification of specific features without changing them explicitly.

Relations to other Patterns: This pattern is closely related to Pattern 3 Resource Change. Depending on the type of the business process the exchange of resources can be a multiplier to increase the impact on the perception of the customers.

\section{Pattern 5: Common Process Improvement}

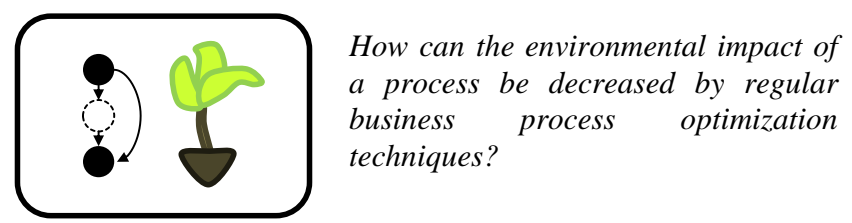

Context: Organizations have widely adopted business process management techniques to manage and optimize their business processes. A wealth of experience is available on optimizing processes with respect to commonly known key performance indicators like time, quality, cost, and flexibility. These experiences may also be applied in order to optimize processes from an ecological perspective.

Challenges: Business process optimization has to deal with a lot of different requirements and constraints. Adding a further dimension to this optimization increases the complexity of the existing trade-off to be solved. The environmental impact may influence the costs, quality, time, or flexibility in positive as well as negative ways.

Solution: The organization identifies potential processes or activities for optimization based on their key performance indicators. They additionally investigate them with respect to their environmental impact and compare them to their strategic objectives.

Sketch:

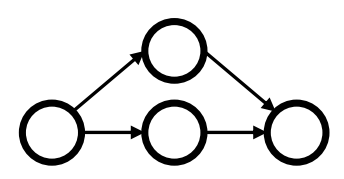

Original Process

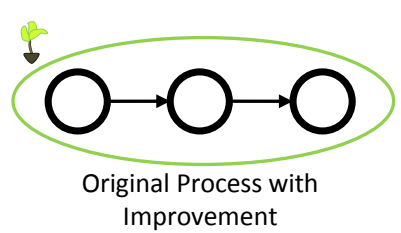

Results: The organization optimizes the processes based on both their key performance indicators and their corresponding environmental impact which decreases the collective environmental impact.

Examples: A commonly known example is the optimization of travel costs for roaming workers within an organization. If the process gets redesigned in such a way that the workers need to travel less, they will entail less fuel consumption which leads to a optimization of the environmental impact. Another example is the following: an organization uses two separate services to perform their calculation of salaries. The first performs the calculation of the monthly working hours of the employees; the second one prepares the cash notes sent to the banking service. All employee information needs to be provided to both services. The organization now replaces those two services by one that determines the cash note directly based on the given information. This common improvement also decreases energy costs for processing and transport of data while shorten the process duration.

Variations: The optimization can be performed concerning any regular key performance indicators, i.e. cost, quality, time, or flexibility. Based on these indicators the optimization method may differ and the impact on the ecological impact varies.

Relations to other Patterns: Depending on the type of business process it may be feasible to utilize automatization or activities performed by human beings as described in Pattern 6 and 7, respectively. These patterns may be applied in addition to aim at regular business process optimization while regarding ecological aspects.

\section{Pattern 6: Process Automatization}

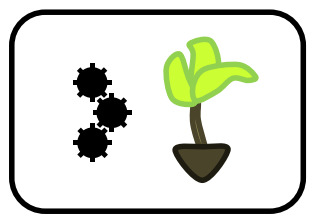

How can the environmental impact of a process be decreased by automating certain activities?

Context: Business processes are based on both human and IT supported activities that aim at achieving a certain strategic objective. These activities need to be organized in such a way that organizations are most profitable and ecological goals within a given environment can be met. Rationalizing some activities is one possibility to reach this objective.

Challenges: The identification of activities that can be automated can be a challenging task. The automated activity needs to perform the task in such a way that either the result remains the same or is even improved or the costs are getting much lower. In the latter case the trade-off between the environmental impact, the costs, and the expected quality needs to be addressed in detail. It must further ensure the global integration with other activities that are dependent on that activity.

Solution: Organizations (partially) streamline specific activities within a business process by automating them. 
Sketch:

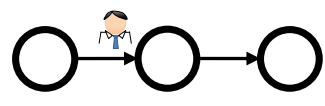

Original Process

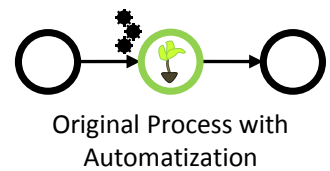

Automatization

Results: The automatization of specific process activities provides the means for optimizations from both, a regular and an ecological perspective. Time, quality, and costs can be improved while energy consumption can be reduced, for example.

Examples: A telecommunication company needs to send its customers an invoice for their connections every month. Usually, the invoices are sent to the customers via mail. Now, the company changes the charging process and transmits the information directly and fully automated to the customers via e-mail. This process restructuring saves time, cost, and in particular decreases the environmental impact caused by the paper manufacturing, the invoice printing, and the transport of the delivery company.

Variations: Single activities must not be totally automated. They can also be semi-automated or split into an automated and a manual part to better (i.e. more efficiently) support human process performance.

Relations to other Patterns: This pattern is strongly related to Pattern 7 (Human Process Performance) as it covers the contrary situation. Depending on the structure of the business process, both patterns can be used to ecologically optimize processes on an activity changing basis. The Patterns 3 and 4 may also tackle the improvement of certain activities in this environment.

\section{Pattern 7: Human Process Performance}

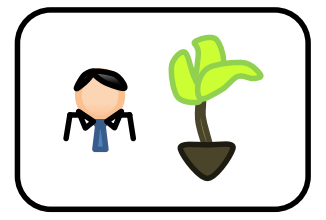

How can the environmental impact of a process be decreased by changing certain activities, so that they are executed by humans?

Context: Organizations are based on both human and IT supported activities and processes that aim to achieve a certain strategic objective. These activities need to be organized in such a way that organizations are most profitable and also reach certain ecological goals within a given environment. Performing some activities by human workers can be one possibility to reach this objective.

Challenges: Determining activities in a workflow which may be replaced by human activities in order to improve the ecological footprint of a certain business process.

Solution: Replace certain activities which are enacted by machines that pollute the environment with human performed activities to obtain a greener business process.

Sketch:

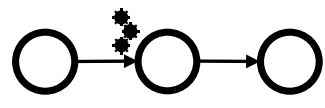

Original Process

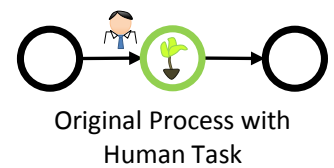

Results: By replacing certain activities of a workflow so that they are enacted by humans, the environmental impact of a business process may be reduced.

Examples: Consider an organization that manufactures specialized metal parts for engineering. The parts are produced in small batch series. Instead of using a high-tech laser-scanning machine which scans each part for quality assurance, an employee checks the corresponding part. This saves the organization costs (at least to some extend) and decreases the environmental impact which is created through the manufacturing and operation of such a specialized machine.

Variations: Single activities must not be executed by humans in total. They can also be semi-automated or split into an automated and a manual part to better (i.e. more efficiently) support human process performance.

Relations to other Patterns: The Automatization pattern (Pattern 6) may be considered as the opposite of the Human Process Performance pattern. Also, the Resources Change pattern (Pattern 3) may be considered because changing an activity to be enacted automatically may be considered the same as using other resources to enact a certain task.

\section{Pattern 8: Outsourcing}

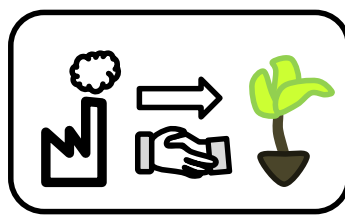

How can the environmental impact of organizations be decreased by de-centralization and outsourcing?

Context: Products and services are usually not provided by just one single organization; instead they are created in collaboration of multiple processes performed by different partners. These choreographies are used to achieve a specific objective more efficiently than one partner could do this on his own. Considering the ecological dimension, this distribution of business processes may also decrease the environmental impact of an organization.

Challenges: Organizations have to consider not only the processes within their own site. On the one hand, the selection of proper business partners comes into focus. On the other hand, it also concerns the improvement of the effective "green footprint" of the complete choreography by splitting the choreography between different partners properly and arranging the overall process interactions.

Solution: One possibility for the improvement of an existing choreography is to swap the business partner(s) to partners offering the same resources or services while meeting current service level agreements but with less ecological impact. However, there is another possibility: making the choreography greener by outsourcing certain activities or processes from the process owner, who is responsible for the process, to another partner providing an equivalent service. By changing one process in the choreography which might have negative impact on the green footprint on its partner's site, another process in the choreography can be made more efficient so that the overall "green footprint" is improved. 


\section{Sketch:}

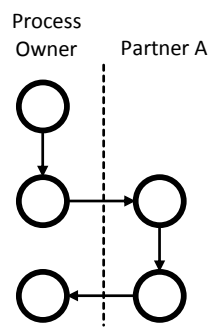

Original Choreography

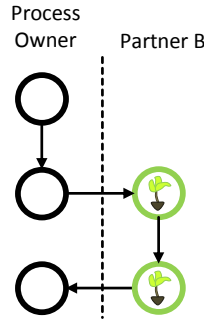

Change Partner

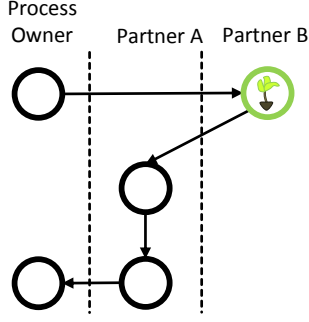

Change Process

Results: Organizations change their current choreographies by either exchanging partners or restructuring the processes involved. Consequently, the environmental impact of an organization is decreased from a global point of view, i.e. based on the optimization of the process choreography between the related partners.

Examples: An example for exchanging the business partner is if partner A decides to use a more ecological supplier for a specific raw material. This could be the use of biodegradable lubricant instead of a chemical one, for example. Another example is the change of the structure of a choreography. Partner $A$ in a choreography may decide to use a software-as-a-service offering from Partner $\mathrm{C}$ for the calculation of salaries that was made inhouse before. On the one hand this reduces the environmental impact on partner A's site, and on the other hand one can assume that due to specialization partner $\mathrm{C}$ performs this task more efficiently than partner A before. This reduces the environmental impact of the complete choreography.

Variations: Instead of permanently changing the structure of a business process or exchanging the business partners, an organization might bind partners dynamically depending on the process request. For example, if a customer wants a green shipment, another shipment partner is used instead of the current shipment partner.

Relations to other Patterns: Depending on the type of business process it may also be feasible to introduce a green business process variant depicted in Pattern 2 or to compensate the environmental impact of the choreography (Pattern 1). Due to the possible restructuring of business processes, Patterns 3 to 7 might also be taken into consideration either stand-alone or as combination to further decrease the environmental impact.

\section{Pattern 9: Insourcing}

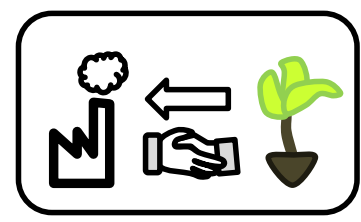

How can the environmental impact of organizations be decreased by centralization?

Context: Products and services are usually not provided by just one single partner; instead they are created in collaboration of multiple processes of different partners. Theses choreographies are used to achieve a specific objective more efficiently than one partner could do it on its own. However, in some cases a centralization of certain activities or processes can influence the global environmental impact of the business process positively.

Challenges: The integration of outsourced processes often requires a significant restructuring of the corresponding processes of a choreography. They need to be integrated on different aspects: the functional layer on the one hand and to assure a more efficient performance of the process from a global point of view at the other hand.

Solution: An organization that utilizes services or processes from third parties may integrate them either in-house or to a special solution provider like a cloud provider, for example.

\section{Sketch:}

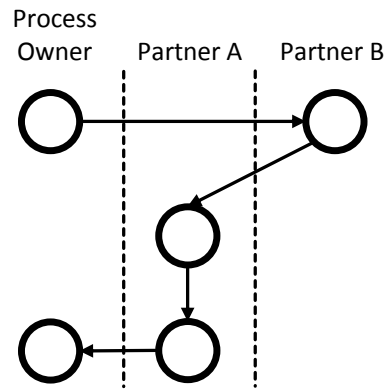

Original Choreography

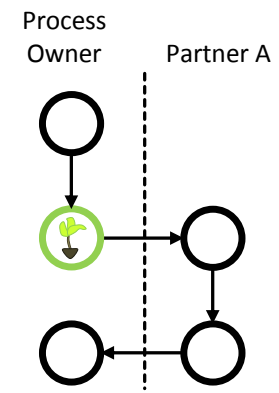

Insourcing Choreography
Results: The centralization of processes or activities within a choreography may provide advantages like economies of scale or reducing logistical efforts when performing certain process steps or activities. This results in providing fewer resources, for example.

Examples: A European car manufacturer produces parts for its cars at a supplier in Asia. To assemble the cars the parts have to be shipped back to Europe either by plane or by ship. The transportation of the parts increases the ecological footprint of each manufactured car a lot. To reduce this footprint the car manufacturer can either insource the production of the parts into his production processes (process change) or he can replace the Asian supplier with a European supplier (partner change).

Variations: Instead of permanently changing the structure of a business process or exchanging the business partners, an organization might bind partners dynamically depending on the process request. In some cases, for example, it might be more efficient to perform a specific activity in-house instead of performing it at the partner's site.

Relations to other Patterns: This pattern is strongly related to the Outsourcing pattern (Pattern 8). Depending on the type of business process the exchange of business partners may also be a feasible solution.

\section{Green Pattern Classification}

The patterns introduced in this paper provide different possibilities to decrease the environmental impact of activities, processes, and organizations as a whole. Depending on how extensively the structure of the business processes can be modified, different patterns may be applied. Consequently, we provide a decision tree that helps organizations to identify patterns fitting their requirements. This decision tree is depicted in Figure 2. We distinguish three major design decisions that need to be made. The first one asks if the process is changeable at all, i.e. whether it is possible to restructure activities or exchange certain 
activities or resources. The second decision considers if the structure of the process is changeable in general or if only the resources it uses and consumes may be exchanged. The third decision asks about the scope of the restructuring, i.e. whether the process to be improved is a stand-alone entity, or if multiple business partners and processes are involved.

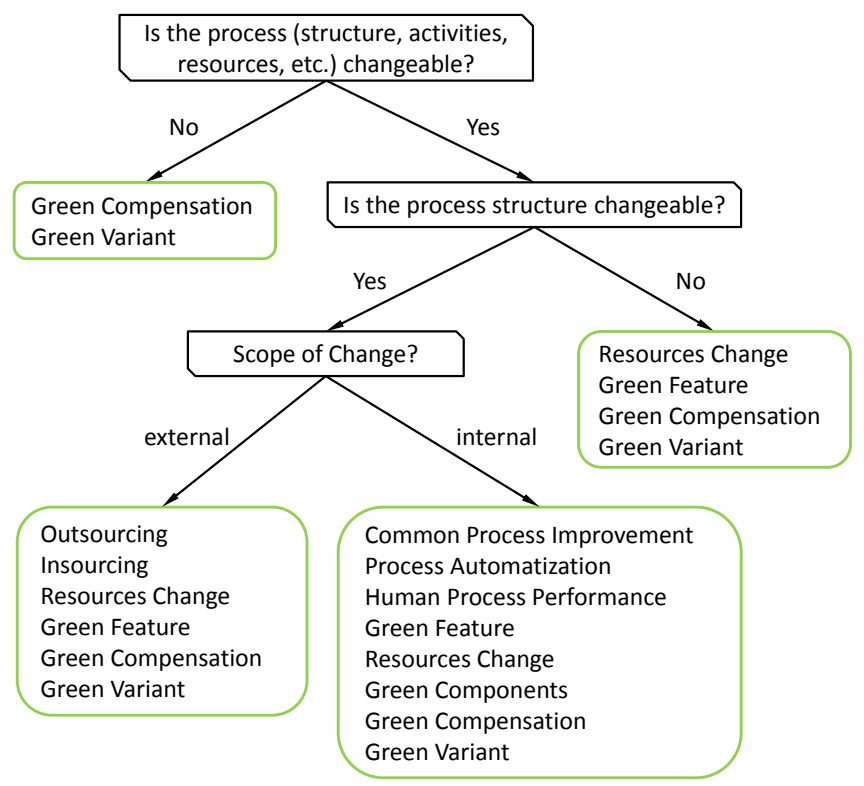

Figure 2 Decision Tree for Green Business Process Patterns

The leafs of the decision tree in Figure 2 indicate the patterns that may be used based on the internal conditions that hold through the decision tree. If a business process of an organization cannot be modified per se, the organization could use the Green Compensation or Green Variant pattern, for example. Otherwise it may apply a pattern that changes the process but not its structure, e.g., by changing the resources utilized by a process. The deeper one dives into the decision tree, i.e. the more the process structure can be changed, the more alternatives are given. These alternatives can be combined in a flexible and arbitrary manner in order to provide the means for different requirements. Again, we do not claim that this classification is complete. Moreover, this provides a starting point that can be extended to cover further requirements.

The re-structuring of business processes with respect to their environmental impact also influences different aspects relevant for competition. In order to support decision makers with choosing appropriate green business process patterns we defined a classification table that contrasts the proposed patterns with different business-related aspects. Thus, the classification, depicted in Figure 3, addresses the trade-off between economic and ecological aspects in an abstract way. Consequently, we have defined the following aspects: The Process / Resource / Process structure changed aspects deal with the grade of restructuring of a certain business process. This aspects are similar to the diversification used within the decision tree. The Used in BPM column indicates whether the optimization method is also be used in regular BPM. The next column, Customers Choice, describes whether customers have the ability to choose between the regular and the optimized business process. Next, the columns about cost stability indicate who is in charge for additional costs occurred from the optimization: the organization or the customer. The last three columns refer to regular key performance indicators, namely time, flexibility, and quality and how they get influenced by the application of the patterns. We chose those aspects in order to provide a general and abstract overview on how organizations may be influenced by applying the proposed patterns. For concrete implementations, however, decision makers need to further analyze the aspects with respect to the concrete situation within their organization.

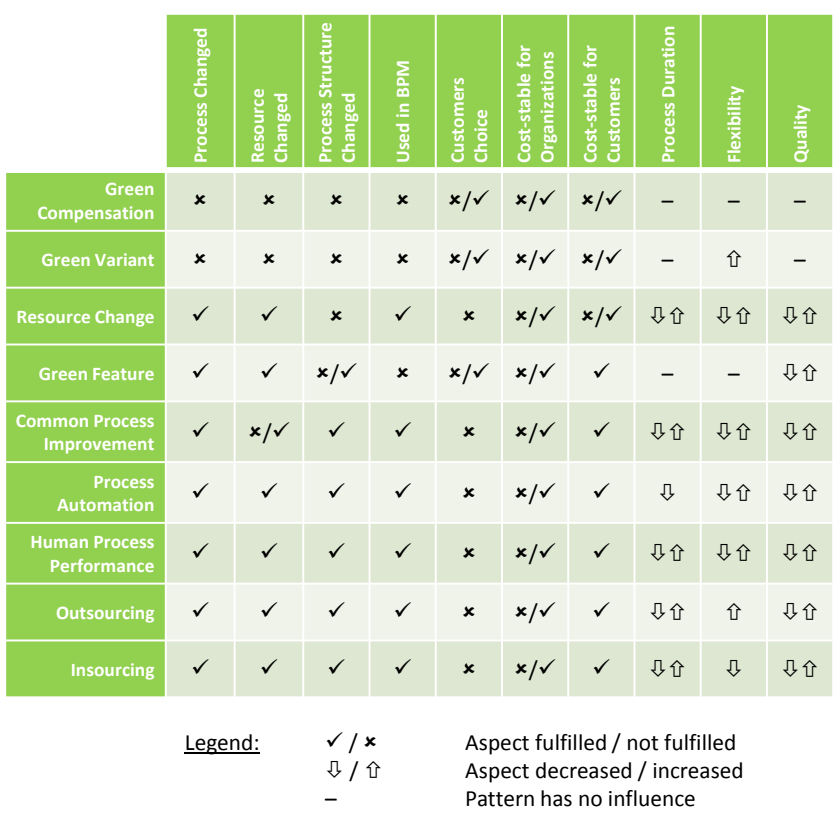

Figure 3 Classification of Green Process Patterns

The terminology of the symbols in Figure 3 is the following: a check or a cross indicates that the given aspect is fulfilled by the corresponding pattern or not, respectively. A process, for example, is not changed in any way by the Green Compensation pattern while the Resource Change pattern does. In some cases we have a check and a cross. Here, the decision depends on the specific situation in which the pattern will be applied. If the Common Process Improvement pattern is applied, for example, it depends on the type and range of process restructuring, the concrete implementation, and whether resources are changed or not. The up and down arrows in the last three columns indicate whether the regular key performance indicators time, flexibility, and quality are influenced positively or negatively. Again, in some cases this is dependent on the concrete implementation of the pattern. We indicated such situations with both an up and down arrow. When applying the Outsourcing pattern, for example, it depends on the corresponding partner and the design of the choreography if the process duration will be decreased or increased.

\section{CRITICAL DISCUSSION}

The patterns proposed in this paper are commonly used within different organizations. However, in some cases the usage of these patterns is disputable. Green Features (Pattern 4), for example, are one of the most critical patterns in this respect. One can hardly distinguish, from an outside perspective, between real business 
process optimization with an environmental focus and so-called "greenwashing". Organizations addressing the latter one only focus on how the appearance of their company or products and services can be improved toward the customers. One could argue that they do not really care about the real environmental impact which makes the "optimization" just a marketing measure. Such behavior also calls the use of certificates or the collaboration with environmental organizations in question because in some cases, environmental organizations are only used as "instruments for industry". Some critics argue that they get sponsored for certifying those organizations' sustainability and eco-friendliness. Consequently, without trustworthy certification authorities and a transparent certification process this course of action needs to be seen with a little precaution.

Following the description of the Green Features pattern there are also approaches combining marketing with real ecological improvement: Volvic Natural Spring Water, for example, reduced the amount of plastic in their bottles by recycling and substituting parts with natural syrup. They do not resign plastic in total, but at least a part of it. This enables, compared to the competitors, a more green reputation. This example shows that a green feature is in fact able to decrease the environmental impact of an organization. However, whether a real improvement or just "greenwashing" is made strongly depends on the management of an organization.

The analysis of current products and offerings showed that Green Compensation (Pattern 1) is one of the most often used green business process pattern in these days. Many companies already set up such compensation processes donating parts of their revenue to ecological projects. This seems to be an easy way to gain customer satisfaction although no real process optimization is performed. Additionally, in most cases actually the customers are charged for the donation to third parties. Often, the pattern is also combined with Green Variants (Pattern 2) to let the customer decide about whether green compensation should be made or not, i.e. the customer pays for compensation. This development shows that organizations are aware of the green change of society, but the conventional strategic objectives still outbalance the green ones. One could argue that organizations want to appear greener as they really are. This trade-off seems to be the biggest barrier in deeply restructuring processes with respect to their environmental impact.

This trade-off issue also affects all other patterns proposed in this paper. Outsourcing (Pattern 8), for example, usually makes more transport necessary. This has to be balanced with the advantages of a greener process at the side of the outsourcing partner. An analysis needs to be done for every concrete scenario. To increase the economic advantages of outsourcing, for example, it would be helpful to collaborate with other organizations (even competitors) that might have the same economic and ecological needs for a certain process step that does not belong to the core competencies of the organization. In this case, the collaboration could provide economic benefit for all parties while achieving a more optimized and environmental friendly process.

\section{CONCLUSION}

In this paper we presented an overview of green business process design patterns concerning the environmental impact of organizations. We identified the solutions of green business process improvements proposed in current research in the field of business process management and Green IT. We also considered green product offerings of companies and identified frequently recurring aspects of their design. We kept the proposed pattern independent from any specific implementation method or process modeling language. So, organizations can apply this knowledge to their existing BPM infrastructure. By proposing these patterns we hope that organizations become aware of their responsibility and critically rethink their current behavior and process design with respect to the environmental impact caused by it.

\section{ACKNOWLEDGMENTS}

Parts of this work were funded by the 7th FP EU-Project S-Cube (Grant Agreement No. 215483). The co-author David Schumm would like to thank the German Research Foundation (DFG) for financial support of the project within the Cluster of Excellence in Simulation Technology (EXC 310/1) at the University of Stuttgart. Many thanks go to our shepherd Ralf Laue for his constructive comments and valuable feedback. Further we want to thank our colleagues at the IAAS for the fruitful discussions.

\section{REFERENCES}

[1] Weske, M. 2007. Business Process Management: Concepts, Languages, Architectures. Springer-Verlag, Berlin Heidelberg.

[2] Hammer, M., and Champy, J. 1994. Reengineering the Corporation: A Manifesto for Business Revolution. Reprint, HarperBusiness.

[3] Ardagna, D., Cappiello, C., Lovera, M., Pernici, B., and Tanelli, M. 2008. Active Energy-Aware Management of Business-Process Based Applications. In Proc. of the 1st European Conference on Towards a Service-Based Internet (ServiceWave '08). Springer-Verlag, Berlin, Heidelberg, 183-195.

[4] Cappiello, C., Ferreira, A., Fugini, M.G., Pernici, B., and Plebani, P. 2010. Application driven IT service management for energy efficiency. In Proc. of SIG Green Workshop at ICIS Conference. Sprouts: Working Papers on Information Systems, 10(131).

[5] Hoesch-Klohe, K., Ghose, A. K., and Le, L.-S. 2010. Towards Green Business Process Management. In Proc. of the IEEE Intl. Services Computing Conference (SCC2010). IEEE Computer Society, Washington, DC, USA, 386-393.

[6] van der Aalst, W. M. P., ter Hofstede, A. H. M., Kiepuszewski, B., and Barros, A. 2003. Workflow Patterns. In Journal of Distributed and Parallel Databases, 14(1). Springer Netherlands, 5-51.

[7] Alexander, C., Ishikawa, S., and Silverstein, M. 1997. A Pattern Language: Towns, Buildings, Construction. Oxford University Press, USA.

[8] Riehle, D., and Züllighoven, H. 1996. Understanding and Using Patterns in Software Development. In Theory and Practice of Object Systems, 2(1). Addison-Wesley Longman, Amsterdam, 3-13.

[9] Deutsche Post AG, Green Logistics. http://www.dhl.com/en/logistics/freight_transportation/go_ green.html

[10] Deutsche Bahn AG, Eco Program. http://www.dbecoprogram.com/index.php?lang=en 
[11] Tchibo direct GmbH, Green Electricity. http://www.tchiboqualitaet.de/content/338386/-

/en/responsibility/environmental-protection-at-

tchibo/climate-protection.html

[12] Firstclimate. http://www.firstclimate.com/

[13] Swiss International Air Lines, my climate. http://swiss.myclimate.org/ and http://www.swiss.com

[14] BASF Corporation, SELECT - Eco Label Manager. https://select-ecolabels.basf.com/Applications/ EcoLabelManager.nsf

[15] Fjällraven, Eco Shell. http://www.fjallraven.com/outdoorequipment/our-fabrics/eco-shell/to-nature-with-love/

[16] Meszaros, G., and Doble, J. 1996. MetaPatterns: A Pattern Language for Pattern Writing. In Proc. of the 3rd European Conference on Pattern Languages of Programming and Computing. The Hillside Group, 4-6.

[17] Leymann, F., and Roller, D. 2000. Production Workflow Concepts and Techniques. Prentice Hall.

[18] Nowak, A., Leymann, F., and Mietzner, R. 2010. Towards Green Business Process Reengineering. In Proc. of the First International Workshop on Services, Energy, \& Ecosystem: SEE2010. San Francisco, USA, 187-192.
[19] Schumm, D., Leymann, F., and Streule, A. 2010. Process Viewing Patterns. In Proc. of the 14th IEEE International EDOC Conference (EDOC 2010). IEEE Computer Society, Washington, DC, USA, 89-98.

[20] Schumm, D., Anstett, T., Leymann, F., and Schleicher, D. 2010. Applicability of Process Viewing Patterns in Business Process Management. In Proc. of the International Workshop on Models and Model-driven Methods for Service Engineering (3M4SE 2010). IEEE Computer Society, Washington, DC, USA, 79-88.

[21] Martin, R. C. 2000. Design Principles and Design Patterns. http://www.objectmentor.com/resources/articles/Principles _and_Patterns.pdf.

[22] Gamma, E., Helm, R., and Johnson, R. 1995. Design Patterns. Elements of Reusable Object-Oriented Software. Addison-Wesley Longman.

[23] Decker, G., Kopp, O., and Barros, A. 2008. An Introduction to Service Choreographies. In: Information Technology. Vol. 50(2). Oldenbourg Verlag.

All links were last followed on May 15, 2011. 